\title{
Canoas en TIERRABomba: TRANSFORMACIONES SOCIOCULTURALES EN LA ISLA
}

\author{
CANOES IN TIERRABOMBA: \\ A SOCIOCULTURAL TRANSFORMATIONS IN THE ISLAND
}

\author{
Por: Margary Martínez Molina* \\ Luis Ángel Puello Orozco**
}

*Estudiante Séptimo

semestre Comunica-

ción Social, Universi-

dad de Cartagena. In-

tegrante semillero de

investigación grupo

Comunicación, Edu-

cación y Cultura.

**Estudiante Séptimo semestre Comunicación Social, Universidad de Cartagena. In-

tegrante semillero de

tegrante semillero de

investigación grupo

Comunicación, Edu-
cación y Cultura

\begin{abstract}
RESUMEN
Este artículo presenta el panorama actual en la isla de Tierra Bomba en Cartagena de Indias tras el proceso de cambio en la construcción de canoas: de la elaboración con madera a la fibra de vidrio y, la importancia social y cultural que esto conlleva. Para ello, utiliza los testimonios de los principales sujetos del proceso: carpinteros, pescadores y líderes sociales cuyos relatos integran la manera cómo este transito afecta sus prácticas culturales.
\end{abstract}

Palabras Clave: canoas, madera, fibra, Tierra Bomba, prácticas culturales.

\begin{abstract}
This article presents the current outlook in Tierra Bomba, an island of Cartagena de Indias, after the process of changes in the construction of the canoes as well as its social and cultural importance. In order to accomplish this work, this paper is based on the testimonies of some of the residents of the island such as carpenters, fishers, and community leaders who can describe the way the transit from wood to fiber affects their cultural practices.
\end{abstract}

Key words: canoes, wood, fiber, Tierra Bomba, cultural practices.

\section{Introducción}

T a isla de Tierrabomba está ubicada en el oeste del mar Caribe y posee aproximadamente 1984 hectáreas de superficie. Se encuentra al norte de la isla de Barú y al sur de Cartagena de Indias: administrativamente pertenece a este distrito. En 1501, la isla fue conocida como isla de Codego. Más tarde, en 1697, como isla de Carex, y por ultimo isla de Tierrabomba en 1741. En la actualidad, está conformada por cuatro centros poblados: Bocachica, Caño del Oro, la vereda de Punta Arena y el poblado de Tierra Bomba que toma el nombre general de toda la isla.

Estos territorios tienen como actividad económica principal, aún hoy, la agricultura (maíz, plátano, caña, melón, batata, patilla, frijol, ajonjolí) y la 


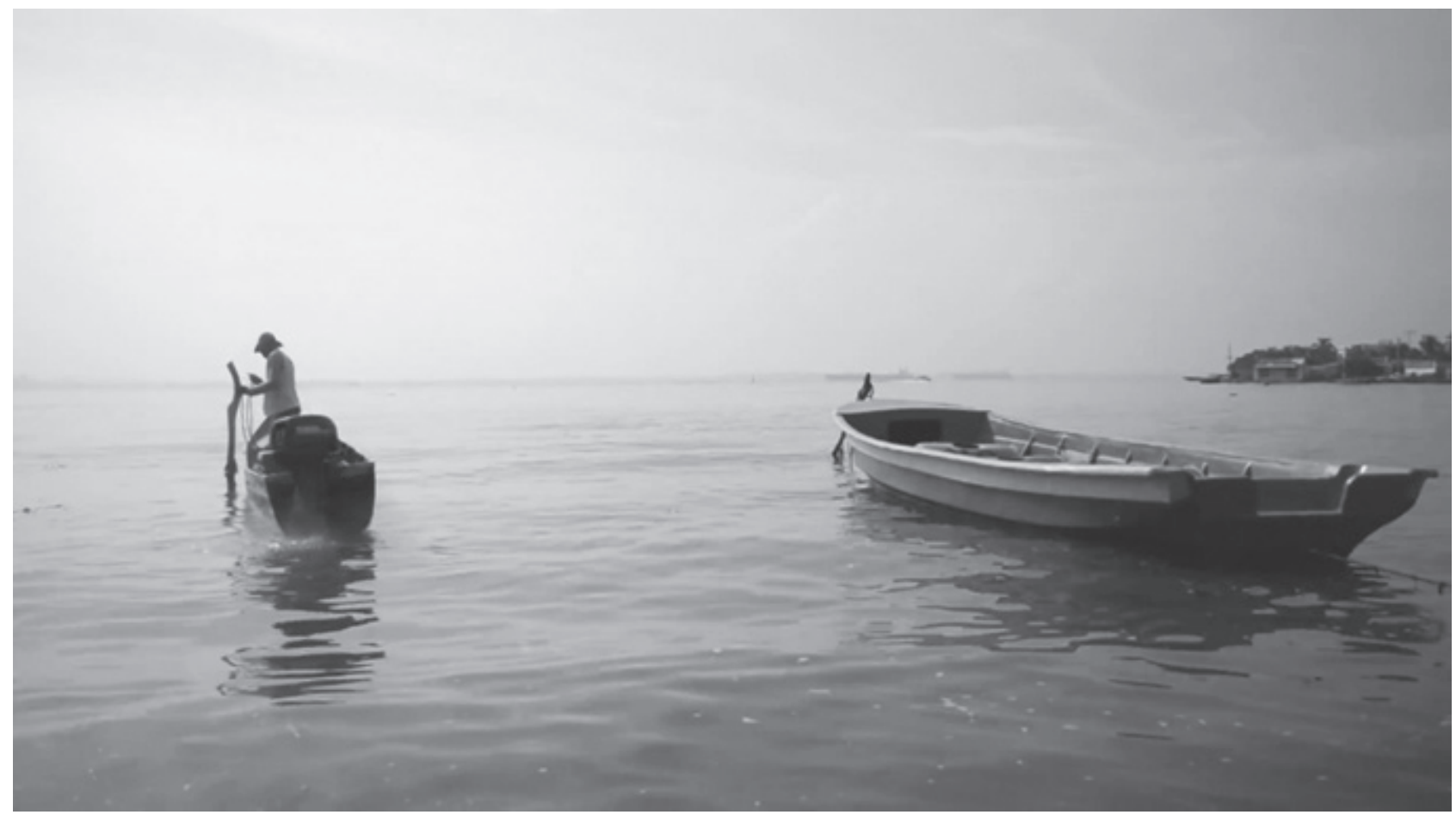

Hombre pescando. Liz Andrea Zarco Quintero, 21 de Noviembre de 2015, Tierra Bomba

pesca. La estrecha relación comercial que sus habitantes han manteniendo con la zona territorial de Cartagena ha configurado un espacio de vida que se relaciona con la tierra y el mar. Este panorama configuró durante mucho tiempo una forma de habitar el territorio que giraba en torno a las embarcaciones artesanales, mayormente conocidas como canoas, pues estas eran el único medio de transporte para llegar a la zona territorial de Cartagena. La construcción de las canoas en madera (manejadas con remos o canaletes) era fuente de sustento para los maestros carpinteros, quienes, a su vez, enseñaban este oficio a sus hijos y nietos.

Las relaciones culturales que la pesca propició se expresaban entre generaciones, sin embargo, la permanencia de la construcción artesanal se vio afectada por algunos cambios, como la implementación de embarcaciones con motor y, principalmente, el uso de la fibra de vidrio para su construcción. Como resultado de este cambio, ciertas prácticas culturales, entendidas estas como ese conjunto de comportamientos, de acciones, de gestos, de enunciados, de expresiones y conversaciones portadoras de un sentido en virtud de los cuales los sujetos de estudio se comunican entre sí y comparten espacios, experiencias representaciones y creencias (Thompson, 1993, págs. 199-212), propias de los isleños que se dedicaban a la construcción de embarcaciones de madera, parecen estar en sus últimas instancias. 
Este ensayo busca describir el proceso de cambio en la construcción de canoas en la isla de Tierrabomba: de la elaboración con madera a la fibra de vidrio y la importancia, social y cultural que este conlleva. Para ello, acude a la información proveniente de un trabajo de campo en el que los datos recolectados, tanto verbal como no verbal, se obtienen de las experiencias dictadas por los protagonistas del fenómeno (pescadores, maestros carpinteros, líderes comunitarios y fibreros), nativos de dos de los cuatro centros poblados de la isla: Bocachica y Caño del Oro respectivamente. De acuerdo al Departamento Administrativo Distrital de Salud de Cartagena (DADIS), los dos centros poblados utilizados para esta reflexión se encuentran ubicados en la Localidad Histórica y Caribe Norte de la ciudad, y están clasificados como corregimientos insulares y rurales.

A su vez, describe el desarrollo y la importancia cultural de las embarcaciones artesanales para los pescadores de la Isla, identifica las principales causantes de la transformación en la elaboración de las embarcaciones y rastrea las nuevas dinámicas creadas por la elaboración de las embarcaciones con fibra de vidrio.

\section{Embarcaciones artesanales: desarrollo e importancia cultural}

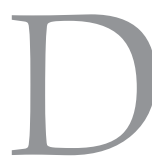

urante la colonia la isla fue el principal punto de llegada de barcos que transportan esclavos provenientes del continente africano (Higuera, 2012), de forma que la configuración identitaria de sus habitantes tiene relación directa con su identificación étnico-racial, esta situación comporta las tareas propias del isleño que se refiere a la pesca artesanal en el Mar Caribe que los rodea como lo hace el líder comunitario Pedro Guerrero: "como comunidad negra es algo que llevamos en la sangre." Además, los habitantes de Tierra Bomba (Tierra Bomba, Caño del Oro, Punta Arena y Bocachica) refieren sus experiencias de manera comunitaria y grupal por lo que se colige que tratándose de una isla, la relación del hombre con el territorio y sus coterráneos es más próxima e íntima.

Las embarcaciones artesanales han hecho y hacen parte de la vida de los habitantes de Tierra Bomba de distintas maneras. En primer lugar, estas se relacionan con actividades económicas puesto que el oficio representaba la fuente de ingreso de todos los carpinteros y de los pescadores y así mismo de los distribuidores de la materia prima. La madera utilizada para canoas era traída de la región del rio Atrato, en un recorrido aproximado de 350 kilómetros, por comerciantes que la vendían en el Mercado Público de Cartagena, con lo que formaban una red económica de servicios. 
Miguel Vásquez al referirse a su rutina diaria deja ver una clara estabilidad económica y dependencia diaria de su eficiencia en la pesca:

"Yo me levantaba temprano como a las seis de la mañana, me tiraba al agua en la embarcación de madera, a bucear caracol, y después cuando ya buceaba caracol, partía el caracol (Sic) y me ponía a pescar. Llegaba aquí a las 3 o 4 de la tarde con 15 o 20 mil pesos de pescado."

En las líneas anteriores vemos también unas construcciones sociales manifestadas en la vida cotidiana. La vida se desarrollaba alrededor de la pesca y las canoas, estas representan no solo su fuente de ingreso sino que además integran gran parte de las actividades realizadas durante el día. Calixto Polo al referirse a las costumbres derivadas de la pesca en canoas artesanales comenta:

"Algo que se perdió fue lo muy gracioso que era cuando se vendía algún producto de la comunidad, ya que aquí teníamos una eslogan cuando se vendía: Ya aquí llegó el pescado, y eso ya no se escucha."

El relato de Calixto muestra cómo la pérdida de las tradiciones propias de los habitantes, gracias a la desaparición de la pesca, disminuye las representaciones e ideas compartidas en la comunidad, las cuales conforman su visión del mundo y aportan sentido a su realidad (Magallón, 2012: 63). El oficio de la pesca y construcción de embarcaciones era transmitida de los maestros carpinteros a los niños a manera de herencia cultural, así mismo esta forma parte de su identidad, al estar dentro de sus experiencias de vida compartidas. Eutorio "Colorado" Díaz y Osvaldo Torres ponen de manifiesto unas muestras identitarias que eran transmitidas y aprendidas entre generaciones:

"Yo, mirando cuando estaba pequeño, le tenía mucho amor a las embarcaciones de barco (Sic)...Ellos nos llevaban desde pelaos y nos ponían ahí a ayudar y coge aquí, aguanta aquí y uno se iba dando de cuenta cómo era que se metía el tablón con las medidas. Y uno de chico se iba dando de cuenta." [Eutorio "Colorado" Díaz]

“...Laureano Caicedo, un señor de Bocachica que fue quien me enseñó la carpintería, fue el que me enseñó a mí a trabajar las embarcaciones de madera, eso puede tener como 50 años, más o menos." [Osvaldo Torres]

A partir de estas prácticas, se puede deducir que la construcción de las canoas representaba una actividad de gran importancia para la población pesquera 
y de carpinteros de Tierra Bomba. Estas prácticas, sin embargo, han cedido ante la implementación de los nuevos materiales para construir canoas, los que añadieron una mayor formalización de la labor.

\section{De la madera a la fibra: posibles explicaciones}

$\checkmark$ on base en las entrevistas realizadas, se pueden identificar las siguientes explicaciones para la transformación de las embarcaciones de madera a fibra de vidrio. En este punto, se debe hacer una revisión mucho mayor de tipo historiográfico. Una primera explicación tiene que ver con el tiempo de duración: una embarcación en fibra de vidrio tiene mayor durabilidad que una embarcación construida en madera, como lo dice Eutorio "Colorado" Díaz:

"Esto es eterno (señala un trozo de fibra), esto dura muchos años, entonces, la madera se pudre, se la comen las polillas se vuelve un disparate, en cambio esto no, esto dura mil años"

Otro factor está relacionado con el tiempo de construcción. Así lo deja ver Juan Castro Angulo:

"La están haciendo de fibra porque es más fácil hoy hacer una embarcación de ese material. Para hacer una embarcación de madera que mida entre 5 y 6 metros de largo por dos metros de ancho duran dos a tres meses haciéndola (Sic). Hoy haciéndola en fibra esa misma embarcación está durando 5 o 6 días".

Una tercera razón, tiene que ver con la visión del desarrollo, lo cual se ve claramente en testimonios como el de Adán Herrera al referirse a su primera construcción en fibra de vidrio:

"Eso fue grande, eso fue un viaje a la luna, porque por lo menos descubrí que ya no estaba en la zona de atraso sino de desarrollo, me sentí en la luna, esa es la gran verdad."

Adán Herrera fue el pescador que inició el tránsito de la madera a la fibra, cuando después de trabajar en Colón (Panamá) constató el "atraso" en que estaban laborando los pescadores y carpinteros de Tierra Bomba. Su testimonio expresa el asombro y el orgullo presente ante la modernización de su trabajo. 


\title{
Dinámicas alrededor de la elaboración de las embarcaciones con fibra de vidrio
}

\begin{abstract}
T os habitantes de la isla de Tierra Bomba reconocen en primer lugar el aporte en términos económicos que la entrada del nuevo material I $\_$representa. La experiencia de todos coincide en el testimonio de que la construcción de las embarcaciones con fibra de vidrio supone una ventaja práctica puesto que, estas tienen una mayor duración, un menor tiempo de construcción, una mano de obra más barata y además no son tan susceptibles al ambiente climático de la isla.
\end{abstract}

Empero, existe, por otro lado, una preocupación latente frente a las prácticas culturales que la nueva tendencia genera. Los habitantes de la isla reconocen la construcción artesanal con madera como una tradición propia que a su vez significaba una herencia generacional. Al aparecer la fibra de vidrio, toda esta tradición de años prácticamente ha desaparecido. Siendo así, las dinámicas ahora establecidas difieren en gran medida de las del pasado. Pedro Guerrero, líder comunitario de Bocachica manifiesta su posición como sigue:

"No es que nos opongamos al desarrollo, nadie se debe oponer al desarrollo; pero sí debemos empoderar a la comunidad, sensibilizarla a que no dejen este trabajo a un lado, y que el desarrollo no pase por encima de la cultura. El desarrollo y la cultura deben asociarse."

La característica más importante del nuevo panorama es la mínima cantidad de personas que siguen dedicándose a la construcción de canoas de madera, puesto que la demanda de estas es casi inexistente. En su lugar, existen microempresas que se dedican a la producción rápida con fibra que cuentan con trabajadores permanentes y con unas labores definidas. En la isla de Caño del Oro existen dos de estas organizaciones, según cuenta Calixto Polo, líder de la isla:

"Hay dos talleres de fibra aquí, y todavía aún se conservan.... El porcentaje de carpinterías es mínimo pero todavía se conservan carpinteros y sitios, que es casi en cualquier orilla.... En cualquier sitio de la playa la persona sube su embarcación y allí se hace su proceso de recuperación de la nave."

Estos dos testimonios permiten construir una nueva versión uniforme de lo que parece ser el escollo generado por el nuevo elemento: la preocupación por la conservación de la memoria cultural de la comunidad frente a los 
beneficios de la fibra. Tanto así que en la actualidad se tiene proyectada la implementación de proyectos que resalten las embarcaciones de madera como elemento central de la cultura isleña, y a su vez sean una opción que potencie el turismo, además la enseñanza de la construcción en madera a los menores para que estos mantengan lo que se considera un legado.

\section{Conclusión}

T a isla de Tierra Bomba en Cartagena de Indias, por su carácter insular, cuenta con un trascender histórico ligado al mar y con ello a la pesca

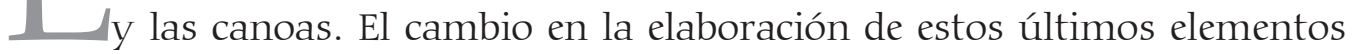
significa para algunos de sus habitantes un llamado de atención sobre la necesidad de mantener vivos los vínculos comunes que conforman su identidad, aun cuando la cultura latinoamericana está constituida de manera fundamental por lo tradicional y lo popular, y la modernización no implica su sustitución (Rodríguez, 2004: 244).

La aparición de la fibra de vidrio significó una formalización del oficio de construcción, debido, sobre todo, a sus beneficios económicos y comerciales. El panorama actual no permite la elaboración artesanal de canoas, pues esta no cuenta con una demanda y resulta de difícil ejecución. La preocupación frente a este hecho ha llevado a que se piensen diferentes iniciativas para la conservación de la tradición de la pesca artesanal como lo es el trabajo con los niños por parte de los maestros carpinteros y un proyecto de construcción de galeones, el cual, de ejecutarse, vincularía a los trabajadores de la isla y a su vez aumentaría el potencial turístico.

El cambio tecnológico puede tornarse vital cuando ocurre en comunidades con tradiciones culturales ancladas a su quehacer en la vida social. La construcción de canoas artesanales en la isla de Tierra Bomba (Tierra Bomba, Caño del Oro, Punta Arena y Bocachica) convoca a la discusión sobre lo tradicional, su rol en la manutención de la esencia histórica social de una comunidad, y la manera como estas deben entenderse en la sociedad contemporánea.

\section{BIBLIOGRAFÍA}

Departamento Administrativo Distrital de Salud, DADIS, (2007). Comportamiento de los eventos bajo vigilancia epidemiológica. Cartagena. Recuperado el 7 de noviembre de 2015 de: https://juanfe.org/wp-content/uploads/2013/03/Epidemiologia-Cartagena-2007.pdf

Higuera, S. (2012). Reflexiones sobre patrimonio cultural. Isla de Tierra Bomba: Lugar en la historia con alta significación cultural. Recuperado el 10 de noviembre de 2015 de: http://fundacionsaurio. blogspot.com.co/2012/04/isla-de-tierrabomba-lugar-en-la.html 
Magallón, M. (2012). "Cultura, tradición y modernidad" En: La Colmena 75 (págs.59-66). Recuperado el 15 de noviembre de 2015 de: http://www.uaemex.mx/plin/colmena/Colmena_75/ Dossier\%20de\%20Filosofia/Cultura_tradicioon_modernidad_Latinoamerica.pdf

Rodríguez, F. (2004). "Modernidad e identidad cultural en América Latina". En: Artes y letras (págs.. 237-255) Recuperado el 15 de noviembre de 2015 de: http://revistas.ucr.ac.cr/index.php/ kanina/article/viewFile/4735/4549

Thompson, J. (1993). Ideolodía y cultura moderna (pdf ed.). (G. F. Caviedes, Trad.) México D.F. : Universidad Autónoma Metropolitana, Unidad Xochimilco. 